\title{
Measles vaccination coverage in high-incidence areas of the Western Cape, following the mass vaccination campaign
}

\author{
G L Bernhardt, N A Cameron, B Willems, A Boulle, D Coetzee \\ Western Cape Government: Health, and School of Public Health and Family Medicine, University of Cape Town \\ G L Bernhardt, FCPHM
}

Division of Community Health, Stellenbosch University, Tygerberg, Western Cape

$\mathrm{N}$ A Cameron, FCPHM

B Willems, MB ChB

Centre for Infectious Disease Epidemiology and Research, University of Cape Town

A Boulle, FCPHM, PhD

D Coetzee, FCPHM

Corresponding author: G L Bernhardt (ginably@gmail.com)

\begin{abstract}
Background. Despite significant advances in measles control, large epidemics occurred in many African countries in 2009 - 2011, including South Africa. South Africa's control strategy includes mass vaccination campaigns about every 4 years, the last of which was conducted nationally in April 2010 and coincided with the epidemic.

Aim. A community survey was conducted in the Western Cape to assess measles vaccination coverage attained by routine and campaign services, in children aged 6 to 59 months at the time of the mass campaign, from high-incidence areas.

Methods. Households were consecutively sampled in high-incidence areas identified using measles epidemic surveillance data. A caregiver history of campaign vaccination and routine vaccination status from the child's Road to Health card were collected. Pre- and post-campaign immunity was estimated by analytical methods.

Results. Of 8332 households visited, there was no response at 3435 (41.2\%); 95.1\% (1 711/1 800) of eligible households participated; and 91.2\% (1 448/1 587; 95\% confidence interval 86 - 94\%) of children received a campaign vaccination. Before the campaign, 33.0\% (103/312) of 9 - 17-month-olds had not received a measles vaccination, and this was reduced to 4.5\% (14/312) after the campaign. Of the 1587 children, $61.5 \%$ were estimated to have measles immunity before the campaign, and this increased to $94.0 \%$ after the campaign .

Discussion. Routine services had failed to achieve adequate herd immunity in areas with suspected highly mobile populations. Mass campaigns in such areas in the Western Cape significantly increased coverage. Extra vigilance is required to monitor and sustain adequate coverage in these areas.
\end{abstract}

S Afr Med J 2013;103(3):181-186. DOI:10.7196/SAMJ.6196

Measles is one of the most infectious human viruses and a significant cause of childhood mortality. Population immunity of around 95\% is necessary to prevent ongoing virus transmission. ${ }^{[1]}$ Significant advances have been made towards measles elimination through a cost-effective vaccine. The World Health Organization (WHO) strategy for reducing measles mortality ${ }^{[2]}$ has been implemented by most WHO member states, including South Africa since 1996. This consists of four arms: (i) achieving sustained high routine first-dose vaccination coverage to infants; (ii) providing a second opportunity for measles vaccination for all children through routine services or mass campaigns; (iii) surveillance of measles and vaccination coverage; and (iv) improving management of complicated cases. The South African childhood schedule recommends routine measles vaccination at 9 months and 18 months of age.

Non-selective mass vaccination campaigns (MVCs) that vaccinate all children in a specified target age group regardless of prior vaccination status have increased coverage, particularly in developing countries where routine health services are weak. ${ }^{[3]}$ MVCs reach children who were not reached by routine programmes, ${ }^{[4]}$ provide a second opportunity for those who did not initially seroconvert, and increase population immunity rapidly, potentially interrupting ongoing transmission. ${ }^{[3]}$ The worldwide impact of MVCs on the incidence of measles has been documented, ${ }^{[1]}$ and revised WHO policy includes MVCs as an outbreak response activity. ${ }^{[2]}$

Despite considerable progress towards measles elimination, epidemics have recently occurred in 28 sub-Saharan African countries, including South Africa. In 2009/2010 approximately 200000 cases were reported in sub-Saharan Africa, ${ }^{[5]}$ of which over 18000 confirmed cases were in South Africa. ${ }^{[6]}$ The underlying cause of these outbreaks is primarily insufficient coverage, but also logistics, including cold-chain maintenance. ${ }^{[5]}$

The South African epidemic started in the Gauteng province in March 2009 and, despite targeted campaigns from May to October $2009^{[7]}$ in affected areas, spread to the rest of the country. An MVC 
against measles and polio had been planned; however, as a result of the epidemic the targeted age group for measles vaccination was extended from 9 to 59 months, to include children from 6 months to 15 years. This was conducted nationally from 12 to 23 April 2010 .

Over 2000 cases $^{[6]}$ were confirmed in the 5.5 million $^{[8]}$ people residing in the Western Cape province; $85 \%$ occurred in the Metropole district, which houses $65 \%$ of the population. Confirmed cases were underestimated, as laboratory confirmation was halted at the peak of the epidemic (Department of Health, Provincial Government of the Western Cape - routine surveillance data, 2010, unpublished). The Western Cape epidemic started in October 2009 and peaked in March 2010. Over $60 \%$ of patients were under 5 years of age and $29 \%$ were in the 6 - 11-month age group. Of patients in the Metropole district $96 \%$ had not received a dose of measles vaccine or had unknown vaccination status, and the case-fatality ratio in under-5s was 6.9/1 000 (Department of Health, Provincial Government of the Western Cape - routine surveillance data, 2010, unpublished).

Routine administrative monitoring of vaccination coverage is required in all health districts in the country. The mobility of people in South Africa means that official district population denominators, especially in urban areas, tend to be underestimated and the large number of indicators considered essential by the various health programmes undermines accurate counts of vaccinations given. This leads to official coverage figures being overestimated, especially in areas with high population mobility. The Western Cape reported that measles coverage in children $<1$ year of age was $102.8 \%, 99.7 \%$ and $102.8 \%$ for the years $2007-2009 .{ }^{[9]}$ The potential for an outbreak was therefore not anticipated. In line with WHO recommendations the Western Cape Department of Health commissioned a community survey in 2010 after the MVC to monitor routine and campaign coverage. ${ }^{[10]}$ The aim was to assess the MVC effectiveness, particularly in reaching children aged 6 months - 5 years at the time of the campaign, from communities in which the incidence of measles had been high, and to determine predictors of routine vaccination coverage and measles.

\section{Methods}

A cross-sectional design was used and five high-incidence suburbs were purposively selected using provincial surveillance of measles cases. Each suburb includes large sections of informal housing. Field-mapping was conducted within each suburb to identify highincidence areas. All households within the high-incidence areas were consecutively visited and requested to participate in the survey. Within each household one caregiver-child pair was randomly selected. Households where there was no response were not revisited owing to the limited time available. A sample of 246 households per suburb was considered to provide vaccination coverage estimates with an alpha error of 0.05 and an absolute precision of 0.05 , based on a coverage estimate of $80 \%$. A caregiver-child pair was eligible to participate if the child was aged $9-62$ months (equivalent to $6-59$ months at the time of the campaign), and had lived in the Western Cape for 6 months or more.

Caregivers were interviewed using a pre-piloted questionnaire, adapted from a previous coverage survey, ${ }^{[11]}$ and routine vaccination status was documented from the child's Road to Health clinic card (RTHC). Campaign vaccination status was not documented on the child's RTHC, and caregiver history was relied upon. Local community members were recruited and paid a stipend to accompany fieldworkers in order to facilitate safer access to the communities. Fieldworkers consisted of three groups of undergraduate medical students and one group of hired fieldworkers. Fieldwork was conducted from 20 July to 30 August 2010.

Data analysis was done using Stata v11. Routine vaccinations administered during or after the MVC were not included in the analysis, in order to provide a pre- and post-campaign profile. Children without an RTHC were regarded as 'not vaccinated', because routine vaccination status according to caregiver history was considered unreliable. Homogeneity within high-incidence areas was not taken into account, as the study did not aim to obtain an overall estimate for the area. Prevalence estimates were calculated with $95 \%$ confidence intervals (CIs). Associations between independent categorical variables and outcomes were explored using a chi-square test and logistic regression. Timeliness of vaccination was illustrated using a cumulative time-to-event distribution. To facilitate comparability across suburbs for the number of reported barriers to access, this variable was adjusted for sample size per suburb.

Population immunity before and after the MVC was modelled based on estimates of waning maternal antibody protection for unvaccinated children aged 6 - 9 months, ${ }^{[12]}$ immunity following routine and campaign vaccine dose $(\mathrm{s}),{ }^{[13]}$ and immunity following measles infection (assumed complete). The effect of parameter uncertainty on the model estimates was assessed through probabilistic sensitivity analysis in which all parameters were varied simultaneously within the published confidence bounds in a Monte Carlo simulation (1 000 runs). Full details of the model and sensitivity analysis are available from the corresponding author.

Verbal informed consent was obtained from all participant caregivers. The Declaration of Helsinki ${ }^{[14]}$ and the South African Medical Research Council guidelines on ethics for medical research ${ }^{[15]}$ were followed. Ethics approval was obtained from the University of Cape Town's Human Research Ethics Committee (HREC Ref.: 331/2010).

Up to date for age was defined as: children aged $9-17$ months who had at least one routine measles vaccination and children 18 months and older who had at least two routine measles vaccinations according to the RTHC.

\section{Results}

The survey was conducted in Site B Khayelitsha, Philippi, Mbekweni, Delft and Du Noon. Of the 8332 households visited, there was no response at 3435 (41.2\%): 95.1\% (1 711 out of 1 800) of eligible households participated. A total of 1587 completed questionnaires were analysed (Fig. 1).

Of primary caregivers, $96.5 \%$ were women, with a median age of 29 years (range 15 - 86 years), and $93.3 \%$ reported that they were literate. The mean age of the children at the time of the campaign was 2.5 years (95\% CI 2.4 - 2.5) (Table 1). Caregivers reported that 8.9\% (142/1 587) of children had had measles (Table 1), and 89.8\% (106/118) reported that it occurred between December 2009 and July 2010, with a peak in April 2010; 79.7\% (94/118) of cases had occurred before the MVC, with a sharp decline in the incidence rate after the MVC.

Of the caregivers $75.2 \%$ (1 194/1 587) had an RTHC for their child, $21.5 \%$ (341) reported that the RTHC was elsewhere and 3.3\% (52) were not in possession of an RTHC. Caregivers in Delft (82.3\%) and $\mathrm{Du}$ Noon $(85.1 \%)$ were significantly more likely to have a card than those in the other sites (Table 1). Of caregivers without an RTHC, $55.0 \%(216 / 393)$ did not know how many doses of measles vaccine had been administered to their child.

Of children older than 9 months with an RTHC, 79.6\% (862/1 083) were up to date for age for measles vaccination. Significant variability 


\section{RESEARCH}

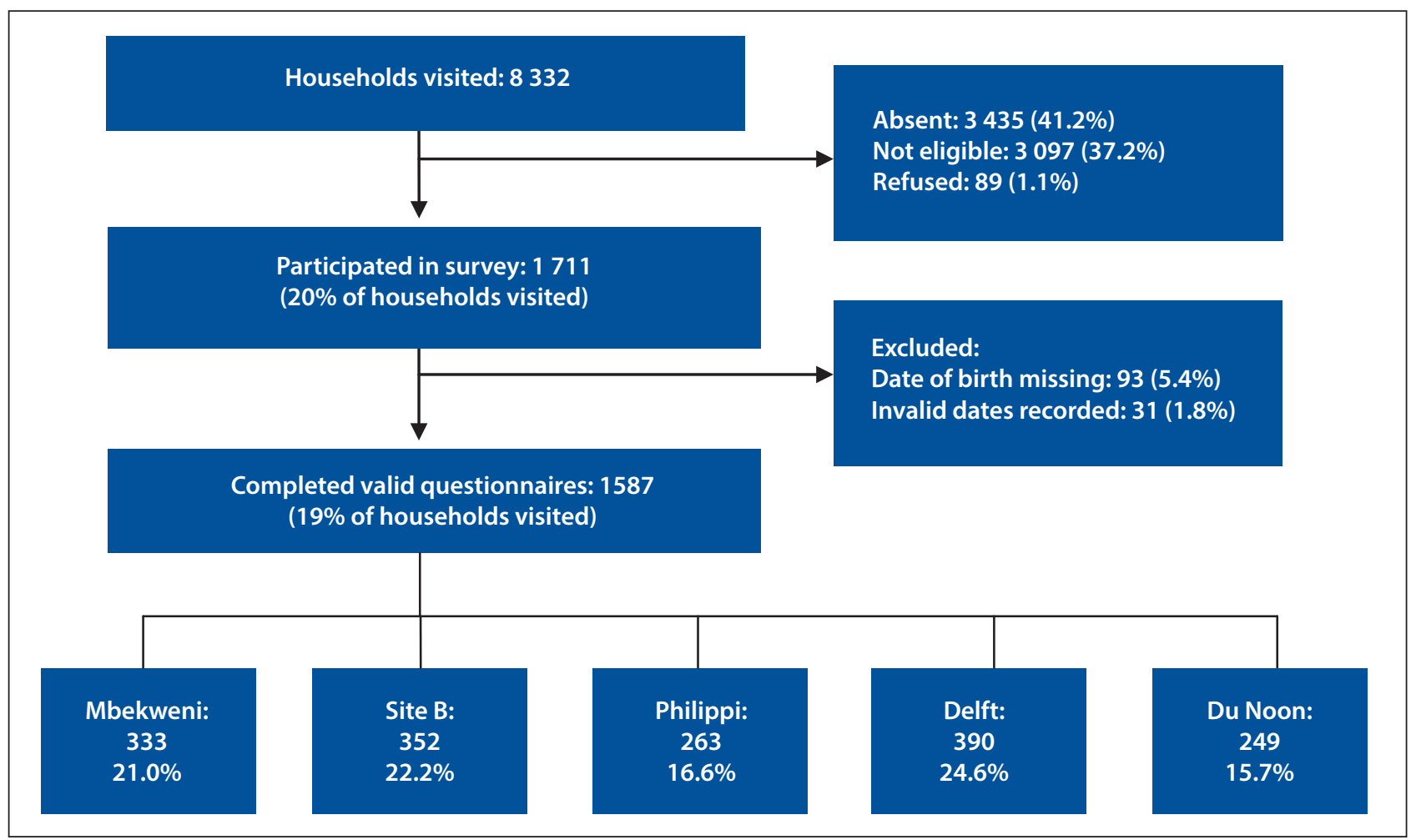

Fig. 1. Selection of study participants.

Table 1. Demographic and measles-related history of study participants

\begin{tabular}{|c|c|c|c|c|c|c|}
\hline & Overall & Mbekweni & Site B & Philippi & Delft & Du Noon \\
\hline Gender of child, $\%$ female & 52.4 & 47.0 & 56.0 & 52.7 & 54.4 & 51.4 \\
\hline$(95 \% \mathrm{CI})$ & $(49.9-54.9)$ & $(41.4-52.4)$ & $(50.8-61.4)$ & $(46.2-58.6)$ & $(49.3-59.6)$ & $(45.2-58.0)$ \\
\hline Age of child (years), mean at time of campaign & 2.5 & 2.6 & 2.6 & 2.4 & 2.4 & 2.4 \\
\hline$(95 \% \mathrm{CI})$ & $(2.4-2.5)$ & $(2.5-2.7)$ & $(2.4-2.7)$ & $(2.2-2.5)$ & $(2.3-2.5)$ & $(2.2-2.5)$ \\
\hline Gender of primary caregiver, $\%$ female & 96.5 & 97.3 & 96.6 & 97.3 & 93.5 & 98.8 \\
\hline$(95 \% \mathrm{CI})$ & $(95.5-97.4)$ & $(94.9-98.8)$ & $(94.1-98.2)$ & $(94.6-98.9)$ & $(90.6-95.8)$ & $(96.5-99.7)$ \\
\hline Age of primary caregiver (years), median & 29 & 30 & 30 & 29 & 29 & 28 \\
\hline (range) & $(15-86)$ & $(15-70)$ & $(17-71)$ & $(15-69)$ & $(16-70)$ & $(17-86)$ \\
\hline Basic literacy of primary caregiver, $\%$ & 93.3 & 90.0 & 94.6 & 96.2 & 94.5 & 91.0 \\
\hline$(95 \% \mathrm{CI})$ & $(91.9-94.4)$ & $(86.0-92.8)$ & $(91.7-96.7)$ & $(93.1-98.2)$ & $(91.5-96.4)$ & $(86.7-94.3)$ \\
\hline History of measles, $\%(n)$ & $9.0(142)$ & $6.0(20)$ & $12.6(44)$ & $10.7(28)$ & $7.4(28)$ & $8.9(22)$ \\
\hline$(95 \% \mathrm{CI})$ & $(7.6-10.5)$ & $(3.7-9.1)$ & $(9.2-16.4)$ & $(7.2-15.0)$ & $(4.8-10.2)$ & $(5.6-13.1)$ \\
\hline History of campaign dose received, \% $(n)$ & $91.2(1448)$ & $93.4(311)$ & $92.0(324)$ & $90.1(237)$ & $90.0(351)$ & $90.4(225)$ \\
\hline$(95 \% \mathrm{CI})$ & $(89.7-92.6)$ & $(90.2-95.8)$ & $(88.7-94.6)$ & $(85.9-93.4)$ & $(86.6-92.8)$ & $(86.0-93.7)$ \\
\hline RTHC seen, \% $(n)$ & $75.2(1$ 194) & $70.0(233)$ & $71.9(253)$ & $66.5(175)$ & $82.3(321)$ & $85.1(212)$ \\
\hline (95\% CI) & $(73.0-77.3)$ & $(64.7-74.8)$ & $(66.9-76.5)$ & $(60.5-72.2)$ & $(78.2-86.0)$ & $(80.1-89.3)$ \\
\hline
\end{tabular}

existed across study sites (Fig. 2). Of all children, 59.5\% (with and without an RTHC) aged 9 months and older before the mass campaign were up to date for age. Significantly more children in the age group $9-17$ months were up to date for age compared with children aged $>18$ months (RTHC subgroup: $85.7 \%$ (95\% CI 80.6 - 89.8) and $77.8 \%$ (95\% CI 74.9 - 80.6\%), respectively). Routine vaccination status was not significantly associated with age and literacy of the primary caregiver, caregiver perceptions of clinic staff, or age and gender of the child.
Date of vaccination was documented for $68.1 \%$ (987/1 449) of those eligible for the first measles dose and 57.4\% (653/1 137) of those eligible for the second measles dose. The median time from the due date to vaccination was 8 days (interquartile range (IQR) 1 - 29) for measles 1 , and 20 days (IQR 1 - 78) for measles 2. Fig. 3 illustrates the cumulative percentage of children given the first and second measles vaccinations, demonstrating delays in the administration of the second dose.

After adjusting for age, documented routine vaccination coverage before the mass campaign was significantly associated with absence 


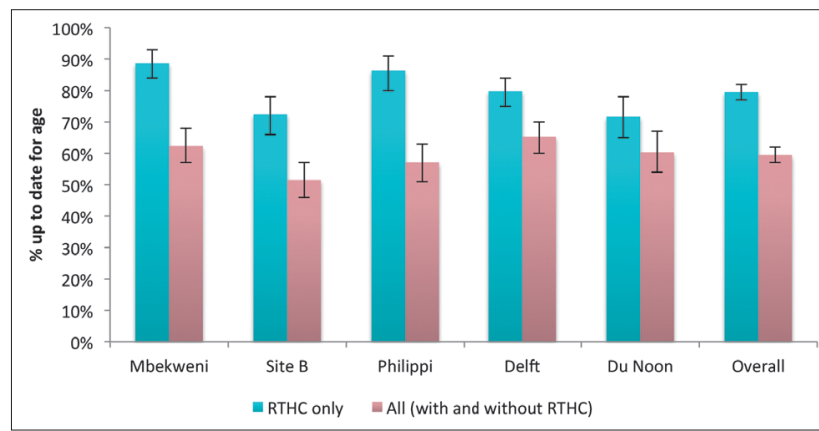

Fig. 2. Proportion of children up to date for age before the MVC, stratified by suburb. Error bars represent $95 \%$ confidence intervals.

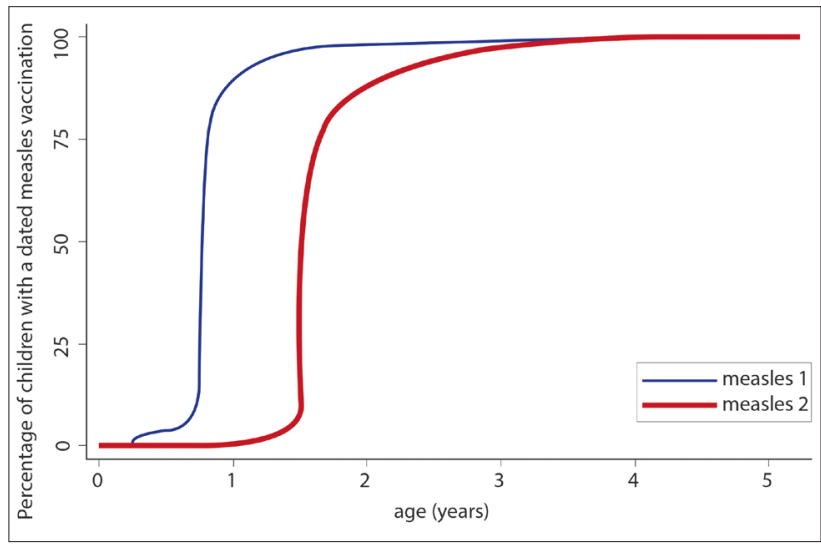

Fig. 3. Timeliness of first and second measles vaccinations in children with date of vaccination documented on the RTHC.

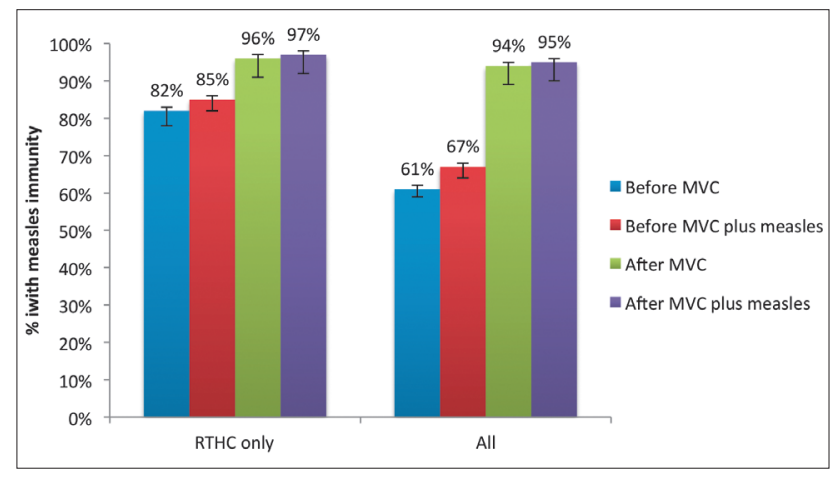

Fig. 4. Estimated measles immunity of participant children. Error bars represent 95\% confidence intervals estimated by Monte-Carlo simulation.

of a history of measles disease in children aged 9 months and older. One measles vaccination was associated with a $49 \%$ reduced odds of contracting measles compared with no measles vaccination $(p=0.003)$, and two measles vaccinations were associated with a $61 \%$ reduced odds of contracting measles compared with no measles vaccination $(p<0.001)$. Two measles vaccinations were associated with a $46 \%$ reduced odds of contracting measles compared with one measles vaccination $(p=0.009)$.

Of caregivers who responded to questions relating to the health services, $85.0 \%$ ( $1238 / 1456)$ reported that the clinic immunisation staff had a positive attitude towards them and their child, $8.2 \%$ $(n=118)$ reported a negative attitude, and 6.9\% $(n=100)$ were neutral. The commonest categories of reasons reported for caregivers not accessing routine vaccination services were related to lack of information $(n=124)$, followed by personal obstacles $(n=99)$ and clinic-related factors $(n=80)$. Lack of information included reasons such as 'unaware of need to return' $(n=41)$, 'unaware of need for vaccination' $(n=30)$ and 'not in area time when vaccination due and unaware that child can go to any clinic for vaccination' $(n=25)$. Reported personal obstacles included reasons such as 'primary caregiver too busy' $(n=44)$, 'family problem' $(n=21)$ and migrationrelated reasons $(n=19)$.

\section{Effectiveness of the mass vaccination campaign}

Caregivers reported that $91.2 \%$ of children (1 448/ 1 587) received a dose of measles vaccine during the MVC. Before the MVC, 33.0\% and $30.1 \%$ of children aged $9-17$ months and 18 months and older, respectively, had not had a dose of measles vaccine, whereas after the MVC this was significantly reduced to $4.5 \%$ and $2.3 \%$, respectively (Table 2).

Immunity from vaccinations administered before the campaign was estimated at $81.6 \%$ for those with an RTHC, and increased to 95.8\% after the campaign. For all children (with and without an RTHC), estimated immunity from vaccination was $61.5 \%$ before and $94.0 \%$ after the mass campaign. The outbreak of measles alone increased immunity by $3.6 \%$ and $5.2 \%$ for those with an RTHC and all children, respectively (Fig. 4).

\section{Discussion}

Routine vaccination services fail to achieve adequate herd immunity in high-risk areas. Caregivers identified lack of information and personal obstacles as the commonest barriers. This finding differs from the 2005 Western Cape provincial vaccination survey, ${ }^{[11]}$ where clinic factors ( $47 \%$ of respondents) followed by lack of information (27\% of respondents) were most frequent. High-risk populations have special access-related needs, and those involved in routine services and mass campaigns need to facilitate access for highrisk populations by more effectively informing communities, and particularly caregivers moving into the area. Community health workers linked to primary care services are suitably placed to facilitate access to health services by monitoring vaccination (and nutrition) status and helping caregivers make sense of the RTHC.

Vaccinations documented on the RTHC were generally administered on time, the second dose less so than the first. Timely vaccination ensures that children are not at risk at an early age. Each time children attend health services represents a chance to vaccinate

Table 2. Proportion of children unvaccinated before and after the MVC, stratified by age group

\begin{tabular}{|c|c|c|c|c|c|c|c|c|}
\hline \multirow[b]{2}{*}{ Portion } & \multicolumn{2}{|c|}{$\begin{array}{l}9 \text { - } 17 \text { months old, } \\
\text { card only }(N=244)\end{array}$} & \multicolumn{2}{|c|}{$\begin{array}{l}9 \text { - } 17 \text { months old, } \\
\text { card plus history }(N=312)\end{array}$} & \multicolumn{2}{|c|}{$\begin{array}{l}18 \text { months and older, } \\
\text { card only }(N=839)\end{array}$} & \multicolumn{2}{|c|}{$\begin{array}{l}18 \text { months and older, } \\
\text { card plus history }(N=1137)\end{array}$} \\
\hline & Before & After & Before & After & Before & After & Before & After \\
\hline With zero doses, $\%(n)$ & $14.3(35)$ & $1(3)$ & $33.0(103)$ & $4.5(14)$ & $5.2(44)$ & $<1(2)$ & $30.1(342)$ & $2.3(26)$ \\
\hline$(95 \% \mathrm{CI})$ & $(10-19)$ & $(0.2-4.0)$ & $(28-39)$ & $(2-7)$ & $(4-7)$ & $(<0.01-0.80)$ & $(2.7-3.3)$ & $(1.5-3.3)$ \\
\hline$\%$ change & - & $91 \%$ reduction & - & $86 \%$ reduction & - & $95 \%$ reduction & - & $92 \%$ reduction \\
\hline
\end{tabular}


them, but the opportunity is often missed. Routine checking of children's RTHCs at every visit, and provision of vaccinations at all health facilities and after-hours, are therefore required.

MVCs can rapidly increase vaccination coverage in high-risk populations, as evidenced by the increase in coverage and the decline in measles incidence after the MVC. Data showed that the outbreak would have continued had it not been for the MVC, as herd immunity would still have been below the required threshold. Earlier implementation of the MVC would have had a greater impact. This is supported by a systematic review by Cairns et al. ${ }^{16}$ that demonstrated a decrease in morbidity associated with rapid outbreak response vaccination.

MVCs have been criticised because they are expensive, logistically challenging and resource intensive. Human resources are usually drawn from routine primary care services, resulting in their interruption and a temporary increase in workload. ${ }^{17}$ However, these campaigns are a necessary intervention, proven to decrease measles morbidity and mortality. ${ }^{18}$ The negative impact on health services can be mitigated by careful planning and conducting the MVC over as few days as is feasible. Campaigns have also had positive spill-over effects on health systems and routine immunisation services, by providing opportunities for better collaboration, improved knowledge of vaccination, micro-planning, data quality and surveillance, and increased community demand for vaccinations. ${ }^{19}$

Vaccination status as documented on RTHCs is often used as a proxy indicator of population immunity. Tools are lacking to interpret outbreak potential in the light of survey data. A risk assessment tool should be developed to model survey data against immunological assays to estimate levels of neutralising antibodies. We estimated population immunity using crude assumptions, and the methodology requires validation and refinement. The absence of recent data describing the decay of maternal antibodies, and the impact of HIV status (maternal and infant), gestational age and maternal measles incidence on the level and attrition of maternally acquired immunity, limit these assumptions. Additionally, the effect of HIV status and vaccination practices on vaccine effectiveness was not taken into account.

The surveillance data from the Western Cape epidemic showed a higher incidence of measles in areas with large informal settlements, consistent with data on other outbreaks. Population density combined with an influx of susceptible persons and decreased access to healthcare services synergistically increase the risk. ${ }^{20}$ Residential mobility is highly prevalent in informal settlements, ${ }^{21}$ is a determinant of non-availability of RTHCs, ${ }^{21}$ and acts as an access barrier to healthcare. Sustainability of the impact of MVCs in informal settlements may be limited. A national MVC evaluation in Khayelitsha found a precipitous drop in vaccination status of immigrants to pre-campaign levels 6 months after an MVC. ${ }^{22}$

Large coverage surveys at a provincial or district level fail to identify specific high-risk areas with low coverage. Administrative coverage estimates are also poor and do not account for in-migration in rapidly expanding areas. Lot quality sampling may be used to identify these high-risk areas, and periodic surveys and mop-up vaccination campaigns should be conducted. In keeping with the vision of the National Department of Health to revitalise primary healthcare, community health workers are ideally placed to strengthen immunisation services and could monitor coverage in high-risk areas and facilitate access to routine services.

\section{Limitations}

A notable limitation of this study was non-response (41.2\%). Children attending crèches were also not included. Those who had resided in the Western Cape for less than 6 months were also considered ineligible, as the study assessed the effectiveness of the campaign in reaching those resident at the time of the campaign. Coverage estimates may be overestimated if those selected were more likely to be vaccinated than those who were absent. Areas that were known to be volatile were avoided, and it is likely that their coverage is lower than elsewhere. Vaccination by the MVC is likely to have been overestimated owing to recall and social desirability bias. Caregiver knowledge regarding the vaccination schedule was limited, and routine coverage in the whole study population was therefore underestimated as children without an RTHC were classified as unvaccinated. Socio-economic and mobility data were not collected, which represents a limitation.

\section{Recommendations}

A proactive approach is required to prevent future measles outbreaks. Although a sensitive surveillance system is in place to identify outbreaks, one case of measles must be considered to be an epidemic and a rapid response should be initiated. Routine services should monitor coverage in high-risk areas such as informal settlements by conducting regular surveys, and immediate action must be taken in areas where coverage fails to achieve herd immunity. Community health workers and school nurses are suitably placed in the primary healthcare re-engineering to monitor and promote the uptake of vaccination. All health facilities should contribute by ensuring that no opportunity for vaccination is missed. MVCs are an important strategy in controlling measles, and careful planning is required to ensure that these are used as opportunities for health systems strengthening. Targeted post-MVC surveys are useful for assessing risk and should routinely form part of MVC planning. Further research is required in developing validated rapid risk assessment methodologies.

Funding. We gratefully acknowledge the Western Cape Department of Health for their support in funding this study.

Acknowledgements. We are grateful to staff from the Department of Health and Cape Town City Health who supported this work and the community members who facilitated fieldwork. We thank the medical students from the University of Cape Town and Stellenbosch University who assisted with collecting data, and the Centre for Infectious Disease Epidemiology and Research, University of Cape Town, for assistance with data entry. We also wish to acknowledge the guidance provided by Francesca Little and Leigh Johnson for the data analysis and modelling.

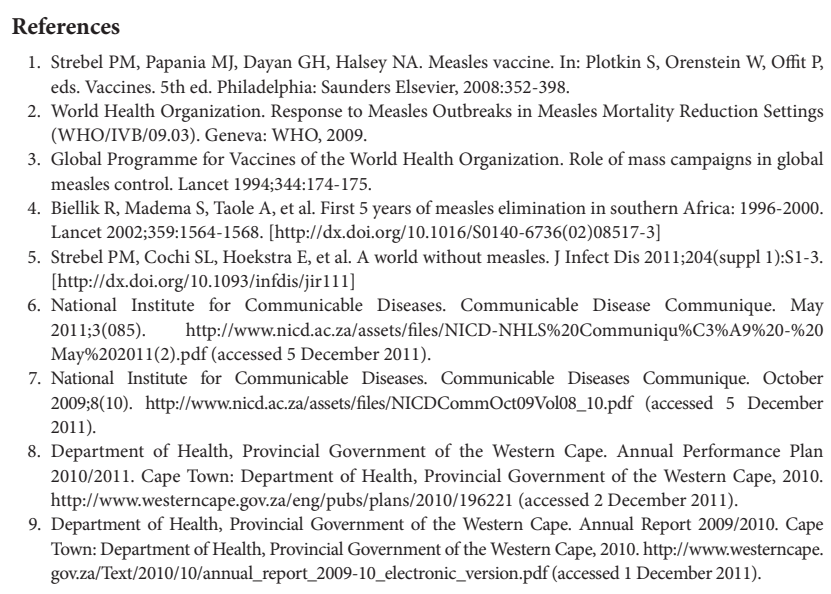

8. Department of Health, Provincial Government of the Western Cape. Annual Performance Plan 2010/2011. Cape Town: Department of Health, Provincial Government of the Western Cape, 2010 2010/2011. Cape Town: Department of Health, Provincial Government of the Western Cape, http://www.westerncape.gov.za/eng/pubs/plans/2010/196221 (accessed 2 December 2011). 9. Department of Health, Provincial Government of the Western Cape. Annual Report 2009/2010. Cape Town: Department of Health, Provincial Government of the Western Cape, 2010. http://www.westerncape.
gov.za/Text/2010/10/annual_report_2009-10_electronic_version.pdf (accessed I December 2011). 


\section{RESEARCH}

10. World Health Organization Regional Office for Africa. Measles SIAs Field Guide. World Health Organization Regional Office for Africa, 2006. http://www.afro.who.int/index.php?option=com_con 2011)

11. Corrigall J, Coetzee D, Cameron N. Is the Western Cape at risk of an outbreak of preventable childhood diseases? Lessons from an evaluation of routine immunisation coverage. S Afr Med

12. Kiepiela P, Coovadia HM, Loening WE, Coward P, Abdool Karim SS. Loss of maternal measle antibody in black South African infants in the first year of life - implications for age of vaccination.

13. World Health Organization, Department of Immunization, Vaccines and Biologicals. The Immunological Basis for Immunization Series. Module 7: Measles. Update 2009. Geneva: WHO, 2009

4. World Medical Association. World Medical Association Declaration of Helsinki. Ethical Principles for Medical Research Involving Human Subjects. Bull World Health Organ 2001;79(4):373-374.

15. Medical Research Council. Guidelines on Ethics for Medical Research: General Principles Including Research on Children, Vulnerable Groups, International Collaboration and Epidemiology. 2002. http://www.sahealthinfo.org/ethics/ethicsbook1.pdf (accessed 7 December 2011).
16. Cairns KL, Perry RT, Ryman TK, Nandy RK, Grais RF. Should outbreak response immunization be recommended for measles outbreaks in middle- and low-income countries? An update. J Infect D 2011,204(suppl 1).S35-46. [ntp://dx.doi.org/10.1093/intdis/jir072]

7. Hanvoravongchai P, Mounier-Jack S, Oliveira Cruz V, et al. Impact of measles elimination activities on immunization services and health systems: findings from six countries. J Infect Dis 2011;204(supp

18. World Health Organization. Proceedings of the Global Technical Consultation to assess the feasibility of measles eradication, 28-30 July 2010. J Infect Dis 2011;204(suppl 1):S4-13,

9. Christie AS, Gay A. The Measles Initiative: moving toward measles eradication. J Infect D 2011;204(suppl 1):S14-17. [http://dx.doi.org/10.1093/infdis/jir075]

20. Kearney M, Yach D, Van Dyk H, Fisher SA. Evaluation of a mass measles immunisation campaign in rapidly growing peri-urban area. S Afr Med J 1989;76(4):157-159.

21. Coetzee N, Yach D, Blignaut R, Fisher SA. Measles vaccination coverage and its determinants in rapidly growing peri-urban area. S Afr Med J 1990;78(12):733-737.

22. Berry DJ, Yach D, Hennink MH. An evaluation of the national measles vaccination campaign in the new shanty areas of Khayelitsha. S Afr Med J 1991;79(8):433-436.

Accepted 13 November 2012. 